\title{
Circadian Hygiene in the ICU Environment (CHIE) study
}

Rob Boots, Gabrielle Mead, Oliver Rawashdeh, Judith Bellapart, Shane Townsend, Jenny Paratz, Nicholas Garner, Pierre Clement and David Oddy, on behalf of the Circadian Investigators in Critical Illness

www.doi.org/10.51893/2020.4.oa9

Published online first 7 December 2020

Human physiology and behaviour typically follow a rhythmic pattern with a period of about 24 hours, and include processes such as cardiac and respiratory function, brain wave activity and drug metabolism. The endogenous timekeeping mechanism - the circadian clock - ensures sequencing of physiological processes.

Circadian rhythm disruption (CRD) is well described in critically ill patients, and associated with severity of illness ${ }^{1-3}$ and inflammatory states. ${ }^{4}$ Sleep disruption and medication use also contribute to CRD. ${ }^{2,5-7}$ The ambient environment, architectural engineering and care routines of the intensive care unit (ICU) significantly modify physiological cues, such as light-dark cycles and intermittent feeding needed for synchronising internal circadian rhythms. 1,2,6,8,9 Ambient temperature and humidity (typically controlled for staff comfort rather than a patient's condition), ${ }^{10}$ sound levels consistently above the standards recommended by the World Health Organization ${ }^{11-13}$ and persistent low lighting levels ${ }^{14}$ could all contribute to CRD. In the Circadian Hygiene in the ICU Environment (CHIE) study, we aimed to investigate environmental circadian cues in the ICU and their potential contribution to CRD as assessed by core body temperature (CBT) profiles.

\section{Methods}

We used a prospective period prevalence study design. Over a 30-day period between July and August 2019, two bed areas per day were monitored in a 27-bed tertiary referral ICU. All patients who were expected to remain in the ICU in the same bed area for at least 24 hours, and who were unlikely to be spending significant time outside of the ICU for procedures, were included in the study. Patients needing infection control isolation were included, and compliance with all infection control protocols was maintained.

The bed areas monitored were randomly allocated in a blinded predetermined sequence that was generated using an online random sequence generator (RANDOM.ORG, Dublin, Ireland). If a bed was empty or the patient was due to be discharged on the day of random assignment, the

\begin{abstract}
Objective: To investigate the environment and care in the intensive care unit (ICU) and its relationship to patient circadian temperature disruption.

Design: 30-day, prospective period prevalence study.

Setting: 27-bed tertiary ICU.

Participants: Patients expected to remain in the ICU for at least 24 hours.
\end{abstract}

Main outcome measures: Temperature, relative humidity, light and sound intensity in the ICU; nursing interventions (using the Therapeutic Intervention Scoring System-28); and core body temperature of ICU patients.

Results: Of 28 patients surveyed, 20 (71\%) were mechanically ventilated. Median (interquartile range [IQR]) light intensity peaked at 07:00 at $165(12-1218)$ lux with a trough at 23:00 of $15(12-51)$ lux and was consistently $<100$ lux between 21:00 and 06:00. Peak median (IQR) sound intensity was at 07:00 (62.55 [57.87-68.03] dB) while 58.84 (54.81-64.71) $\mathrm{dB}$ at 02:00. Ambient temperature and humidity varied with median (IQR) peaks of $23.11^{\circ} \mathrm{C}$ $\left(22.74-23.31^{\circ} \mathrm{C}\right)$ at $16: 00$ and $44.07 \%(32.76-51.08 \%)$ at $11: 00$ and median troughs of $22.37^{\circ} \mathrm{C}\left(21.79-22.88^{\circ} \mathrm{C}\right)$ at $05: 00$ and $39.95 \%(31.53-47.95 \%)$ at $14: 00$, respectively. Disturbances to sleep during the night occurred due to care activities including linen changes (15 patients, 54\%) and bathing $(13,46 \%)$. On the day before and the day of the study, 13 patients (47\%) and 10 patients (36\%), respectively, had a circadian rhythm on core body temperature without an association with illness severity, nursing intervention or environmental measures.

Conclusions: The ICU has low light intensity with relative humidity and ambient temperature not aligned to normal human circadian timing. Noise levels are commonly equivalent to conversational speech while patient care procedures interrupt overnight sleep. The contribution of these factors to disrupted CBT rhythmicity is unclear.

Crit Care Resusc 2020; 22 (4): 361-369 


\section{ORIGINAL ARTICLES}

next suitable bed in the schedule was monitored. The beds not monitored in the initial random sequence were later randomly assigned using a new sequence, after assignment of beds using the first random sequence was completed.

Environmental monitoring of each bed area was done using equipment mounted on a purpose-built trolley that was placed at the head of the bed, as close as practicable to the horizontal plane of the patient's eyes. Light intensity, temperature and humidity data were measured using the HOBO U12 external logger (Onset Computer Corporation, Bourne, Mass, USA) calibrated according to the manufacturer's instructions with minutely sampling. The HOBO U12 detects light in the range of between 1 and 3000 lumens, a relative humidity of between $5 \%$ and $95 \%( \pm 2.5 \%)$ and ambient temperature of between $20^{\circ} \mathrm{C}$ and $70^{\circ} \mathrm{C}\left( \pm 0.35^{\circ} \mathrm{C}\right)$. Sound intensity was recorded using the Decibel X PRO (iOS) application with an external Dayton Audio iMM-6 microphone (Dayton Audio, Springboro, Ohio, USA) calibrated using AudioTools (Studio Six Digital, AudioControl, Mountlake Terrace, Wash, USA). Decibel X PRO was used to sample every 0.5 seconds with a range of 20-150 dB. After monitoring each bed area, survey equipment was decontaminated using Clinell wipes (GAMA Healthcare, Watford, Hertfordshire, England).

The principal study variable was the complete 48-hour CBT profile representing the day before, day of and day after the environmental monitoring, and was measured using an indwelling urinary catheter thermistor. Oesophageal, tympanic or axillary temperatures (listed here in order of preference) were included only where bladder temperatures were not available. Patient-centric data to describe the cohort included: Charlson comorbidity score; ${ }^{15}$ principal ICU admission diagnosis (surgical, elective, medical, trauma, obstetric); sepsis requiring antibiotic therapy on admission; APACHE (Acute Physiology and Chronic Health Evaluation) II score ${ }^{16}$ and sepsis-related organ failure assessment (SOFA $)^{17}$ score on admission and on day of environmental monitoring; pharmacological and non-pharmacological interventions including Therapeutic Intervention Scoring System-28 (TISS-28) scores (Online Appendix, table S1); 18 and the timings of nursing interventions and patient activities from data as recorded in the electronic patient records (MetaVision, iMDsoft, Tel Aviv, Israel).

De-identified data were recorded in a purpose-built research database (Access, Microsoft, Redmond, Wash, USA). Hourly mean measurements from each bed area were pooled to create a temporal profile of the unit as a whole over 24 hours. Pooled environmental and clinical data are described as medians and interquartile ranges per hour. Actual data ranges are reported to describe the spread of a broad distribution where relevant.
Pooled environmental data and CBT data were assessed for rhythmicity and the degree of variability using the R-4.0.3 statistical program and $R$ packages cosinor and cosinor 2 to fit a cosine curve of best fit (R Foundation for Statistical Computing, Vienna, Austria). Rhythmicity is reported as per cent rhythm, indicating goodness of fit $\left(r^{2} \times 100\right)$. The midline estimating statistic of rhythm (MESOR) and amplitude of the fitted curve are also reported. Logistic models included variables where associations in univariate analysis had $P \leq 0.25$, and variables considered to have clinical relevance with goodness of fit and calibration assessed by the Hosmer-Lemeshow statistic and area under the receiver-operating characteristics curve, respectively. These analyses were completed using Stata 15.5 statistical software (StataCorp, College Station, Tx, USA).

This study was approved by the Royal Brisbane and Women's Hospital Human Research Ethics Committee (LNR/2019/QRBW/53498). The requirement for written informed consent was waived by the committee because only routinely collected de-identified clinical data were used, and collection of environmental data did not require direct patient participation.

\section{Results}

Over the 30-day period of the survey, 28 patients and their immediate ICU bed environments were studied; the median period of ICU admission on the study day was 5 days (interquartile range [IQR], 2.5-8 days). One patient's bed area was surveyed twice due to difficulties collecting data. About half of the patients were women, and the median age of patients was 60 years (IQR, 26-72 years). The median admission APACHE II and SOFA scores were 24 $(I Q R, 13-28)$ and 8 (IQR, 3-11), respectively. Twenty patients $(71 \%)$ were mechanically ventilated and required nursing interventions, and the median TISS-28 for all patients was 33 (IQR, 27-36) on the day of the study (Table 1). There were 19 medical patients (68\%), one surgical patient (4\%) and eight trauma patients (29\%). The median number of Charlson comorbidities recorded before ICU admission was 2 (IQR, 1-4). The casemix included five patients admitted with sepsis (18\%), four with subarachnoid haemorrhages $(14 \%)$, four multitrauma patients (14\%), three neurotrauma patients $(11 \%)$, two patients who had suffered out-ofhospital cardiac arrests (7\%) and two burns patients (7\%). All 28 patients received some form of sedative - including narcotics (16 patients, 57\%), antidepressants (three, 11\%), major tranquillisers (one, 4\%) and anaesthetic agents $(16,57 \%)$ - on the day before, day of or day after the survey. Antibiotics were prescribed for 15 patients (54\%) on the day of the survey. Detailed drug prescription data are summarised in the Online Appendix (table S2). Nursing interventions and activities are summarised by time of 


\section{ORIGINAL ARTICLES}

Table 1. Patient demographics and summary of cumulative intensive care unit interventions before day of study $(N=28)$

\begin{tabular}{|c|c|}
\hline Parameter & $\begin{array}{c}\text { Median (IQR) [range] or } \\
\text { number }(\%)\end{array}$ \\
\hline Age (years) & $60(26-72)[20-81]$ \\
\hline Sex (female) & $15(53.6 \%)$ \\
\hline \multicolumn{2}{|l|}{ Admission type } \\
\hline Medical & $19(67.9 \%)$ \\
\hline Surgical & $1(3.6 \%)$ \\
\hline Trauma & $8(28.6 \%)$ \\
\hline \multicolumn{2}{|l|}{ Charlson comorbidity score } \\
\hline Sum & $2(0-3)[0-5]$ \\
\hline Count & $2(1-4)[1-5]$ \\
\hline \multicolumn{2}{|l|}{ SOFA score } \\
\hline Day of admission & $8(3-13)[0-15]$ \\
\hline Day before survey & $6(4-10)[1-13]$ \\
\hline Day of survey & $6.5(5-9)[1-15]$ \\
\hline \multicolumn{2}{|l|}{ APACHE II score } \\
\hline Day of admission & $24(13-28)[4-32]$ \\
\hline Day before survey & $21(14-24.5)[1-29]$ \\
\hline Day of survey & $19(14-24)[6-29]$ \\
\hline Mechanical ventilation & $20(71.4 \%)$ \\
\hline Duration (h) & 135 (12.5-191.5) [0-863] \\
\hline Continuous dialysis & $1(3.6 \%)$ \\
\hline Duration (h) & 69 \\
\hline Inotrope use & $16(57.1 \%)$ \\
\hline Duration (h) & $37(5-60.5)[2-212]$ \\
\hline Sedation & $20(71.4 \%)$ \\
\hline Duration (h) & $90(12.5-126.5)[5-470]$ \\
\hline Continuous nasogastric feeding & $16(57.1 \%)$ \\
\hline Duration (h) & $147.4(14.5-182)[1-801]$ \\
\hline \multicolumn{2}{|l|}{ TISS-28 score (all patients) } \\
\hline Day before survey & $33(27-36)[15-42]$ \\
\hline Day of survey & $33(27-36)[15-42]$ \\
\hline
\end{tabular}

APACHE = Acute Physiology and Chronic Health Evaluation. $\mathrm{IQR}=$ interquartile range. SOFA = sepsis-related organ failure assessment. TISS-28 = Therapeutic Intervention Scoring System-28.

day in Table 2. A high proportion of patients experienced significant disturbances to sleep during the night owing to linen changes $(15,54 \%)$ and bathing $(13,46 \%)$.

Overall, the ambient light in the ICU during the day was equivalent to that of a very dark day. Light intensity peaked in the morning between 07:00 and 08:00 with a median (IQR) of 165 (12-1218) lux; it was lowest at 23:00 with a median (IQR) of 15 (12-51) lux and was consistently
$<100$ lux between 21:00 and 06:00. The pooled hourly light intensity data for all bed areas by time of day are summarised in Figure 1 (panel A). Median light intensity differed significantly between bed areas, and ranged from 15.7 lux to 134.4 lux $(P=0.001)$. Light intensity had a rhythmic character (Figure 1, panel B) (rhythmicity, $0.8 \%$; $P<0.0001$ ) with a MESOR of $50.64 \pm 0.37$ lux and an amplitude of 38.68 lux.

The daily median of hourly sound intensity was $59 \mathrm{~dB}$, and the daily median for individual bed areas ranged from $50 \mathrm{~dB}$ to $69 \mathrm{~dB}(P=0.001)$ (Figure 2, panel A). There was no difference in the median (IQR) daily sound intensity between isolation rooms and open-bay bed areas (66 [5467] $\mathrm{dB} v 59$ [57-640] $\mathrm{dB} ; P=0.15$ ). The daily median (IQR) hourly maximal sound intensity was $86(81-90) \mathrm{dB}$. The median (IQR) time where sound intensity was greater than $60 \mathrm{~dB}$ was 48 (15-139) seconds. The hourly median sound intensity did not differ significantly by time of day $(P=0.09)$, although sound intensity had a rhythmic character (Figure 2 , panel $B$; rhythmicity, $68 \% ; P<0.0001$ ) with a MESOR of $60.26 \pm 0.02 \mathrm{~dB}$ and a small amplitude of $1.46 \mathrm{~dB}$.

The median of the mean hourly ambient temperatures of all bed areas across the 24-hour period of measurement was $22.8^{\circ} \mathrm{C}\left(\mathrm{IQR}, 22.4-23.1^{\circ} \mathrm{C}\right)$ with a peak of $23.1^{\circ} \mathrm{C}$ $\left(\mathrm{IQR}, 22.74-23.31^{\circ} \mathrm{C}\right)$ at $16: 00$ and a trough of $22.37^{\circ} \mathrm{C}$ $\left(\mathrm{IQR}, 21.79-22.88^{\circ} \mathrm{C}\right)$ at $05: 00$ (Figure 3, panel A). The median ambient temperature across the day differed significantly between individual bed areas (range, 21.1$\left.26.6^{\circ} \mathrm{C} ; P=0.001\right)$, and this difference remained when burns patients were removed from the analysis $(P<0.001)$. Ambient temperature had daily rhythmicity (Figure 3, panel B) (rhythmicity, $88 \% ; P<0.0001$ ) with a MESOR of $22.8 \pm$ $0.002^{\circ} \mathrm{C}$ and an amplitude of $0.34^{\circ} \mathrm{C}$.

The median of the mean hourly relative humidity for all bed areas across the 24-hour period was 47.1\% (IQR, 29.5$51.8 \%)$ with a peak of $49.7 \%(\mathrm{IQR}, 31.9-53.3 \%)$ at $20: 00$ and a trough of $43.1 \%(I Q R, 31.5-48.5 \%)$ at $14: 00$, and mean hourly relative humidity values ranged from $18.6 \%$ to $73.0 \%$ (Figure 4, panel A). Median daily relative humidity differed significantly across the bed areas, and ranged from $24.5 \%$ to $64.5 \%(P<0.001)$. Relative humidity had daily rhythmicity (Figure 4, panel B) (rhythmicity, 48\%; $P<0.0001$ ) with a MESOR of $43.01 \pm 0.002 \%$ and an amplitude of $1.04 \%$.

The CBT profiles showed that 14 patients (50\%) had a rhythmic temperature profile on both the day of and the day before the survey. For the day of the study, the median of the MESOR was $37.7^{\circ} \mathrm{C}\left(\mathrm{IQR}, 37.3-38.4^{\circ} \mathrm{C}\right)$; median amplitude was $0.22^{\circ} \mathrm{C}\left(\mathrm{IQR}, 0.12-0.20^{\circ} \mathrm{C}\right)$ with a median phase of 17:08 hours (IQR, 05:52-20:11). Using a 2-hour window each side of 18:00 as the time of the normal temperature peak for humans with a rhythmic CBT, 


\section{ORIGINAL ARTICLES}

Table 2. Patient care activities on day of environmental monitoring

\begin{tabular}{|c|c|c|c|}
\hline \multirow[b]{2}{*}{ Activity } & \multicolumn{3}{|c|}{ Median (IQR) [range] or number (\%) } \\
\hline & $\begin{array}{c}\text { Time: } 07: 00- \\
13: 00(n=27)\end{array}$ & $\begin{array}{c}\text { Time: } 13: 00- \\
21: 00(n=28)\end{array}$ & $\begin{array}{c}\text { Time: } 21: 00 \\
07: 00(n=28)\end{array}$ \\
\hline Sit out/mobilise & $6(21.4 \%)$ & $5(18.5 \%)$ & 0 \\
\hline Chest physiotherapy & $9(32.1 \%)$ & $15(55.6 \%)$ & $1(3.6 \%)$ \\
\hline Other physiotherapy & $8(28.6 \%)$ & $15(55.6 \%)$ & $1(3.6 \%)$ \\
\hline Bathing & $6(21.4 \%)$ & $10(37.0 \%)$ & $13(46.4 \%)$ \\
\hline Mouth care & $28(100 \%)$ & $28(100 \%)$ & $28(100 \%)$ \\
\hline Eye care & $23(82.1 \%)$ & $23(85.2 \%)$ & $23(82.1 \%)$ \\
\hline Dressings & $4(14.3 \%)$ & $7(25.9 \%)$ & $7(25.0 \%)$ \\
\hline Linen change & $9(32.1 \%)$ & $13(48.2 \%)$ & $15(53.6 \%)$ \\
\hline $\begin{array}{l}\text { Blood product } \\
\text { administration }\end{array}$ & $5(17.9 \%)$ & $4(14.8 \%)$ & $5(17.9 \%)$ \\
\hline $\begin{array}{l}\text { Non-invasive blood } \\
\text { pressure measurement }\end{array}$ & $1(0-2)[0-5]$ & $2(1-3)[0-8]$ & $1(0-5)[0-8]$ \\
\hline Airway suctioning & $2(0-4)[0-7]$ & $3(0-4)[0-7]$ & $3.5(3-6)[2-8]$ \\
\hline Turns & $3(2-4)[1-5]$ & $3(3-4)[1-5]$ & $4(2-4)[1-5]$ \\
\hline Dialysis therapy & $1(3.6 \%)$ & $1(3.6 \%)$ & $1(3.6 \%)$ \\
\hline Dialysis circuit change & $1(3.6 \%)$ & 0 & 0 \\
\hline Oral feeding & $8(28.6 \%)$ & $9(32.1 \%)$ & $11(39.3 \%)$ \\
\hline $\begin{array}{l}\text { Nasogastric/nasojejunal } \\
\text { feeding }\end{array}$ & $21(75.0 \%)$ & $14(50.0 \%)$ & $18(64.3 \%)$ \\
\hline Total parental nutrition & $1(3.6 \%)$ & 0 & 0 \\
\hline Visitors & $21(75.0 \%)$ & $10(35.7 \%)$ & $8(28.6 \%)$ \\
\hline
\end{tabular}

$\mathrm{IQR}=$ interquartile range.

six patients (43\%) were phase advanced and four (29\%) were phase delayed. Circadian comparisons of temperature data for three ICU patients versus normal temperature profiles are shown in Figure 5. Multiple logistic regression found no associations between CBT rhythmicity and any of the measured environmental factors, illness severity scores or measures of intensity for nursing interventions (Online Appendix, Table S3).

\section{Discussion}

Our results from the CHIE study confirm poor "circadian hygiene" in the ICU environment, with consistently low light levels, persistent noise (commonly at conversational levels, and often higher, across the 24-hour day), and significant variability in temperature and relative humidity measured in different bed areas. ${ }^{11,14,19}$ These parameters are the baseline cues that synchronise circadian clocks of organ systems and metabolic pathways. ${ }^{20,21}$ Previous investigations into the ICU environment have assessed environmental parameters in isolation without attempting to link the findings to patient clinical status. The ICU environment is subject to large variations in ambient conditions owing to differences in hospital and ICU designs and layouts, patient monitoring methods, seasonal variation, engineering plant and servomechanical control systems.

Although the ICU environment in our study was generally dark at night, the daytime lighting was the equivalent of a "dull" day. This finding is similar to those of previous studies, where diurnal variation in light intensity has been shown but with consistently low light levels during the day relative to natural sunlight exposure. ${ }^{14}$ Insufficient light exposure potentially removes or at least weakens the ability of light to function as a zeitgeber for the circadian timing system, including entrainment of melatonin rhythms with minimal melatonin secretion when subjects are exposed to 6.5 hours of light of an intensity below 100 lux, 22 similar to the light levels found in our study. The minimal light intensity to entrain melatonin rhythmicity is not known. Numerous studies have shown a lack of melatonin rhythmicity in ICU patients, 5,23 but the contribution of low light levels to ICU patients with complex disease is unclear.

To our knowledge, no trials have specifically documented the effects of the components of the artificial environment of the ICU on human circadian rhythm apart from associations which may be confounded by the effects of critical illness itself. Recommendations to prescribe appropriate environmental circadian cues have been extrapolated from laboratory research, shift-work studies and small clinical trials. Outdoor illumination is of the order of 5000 to 100000 lux, compared with 50 to 500 lux for artificial indoor light. 24 Published reports on ambient light exposure in the ICU vary significantly. ${ }^{25}$ Despite normalised irradiance, natural and artificial light sources that qualitatively appear white can greatly vary in colour temperature, spectra shape and scale. ${ }^{26}$ Artificial nocturnal light with intensity as low as 40 lux from 1-metre distance, brief duration of exposure (under 5 minutes), evening exposure (more significant than afternoon exposure), blue wavelengths typical of electronic devices and daylight white colour temperature (6000 K) all have significant effects on melatonin suppression and circadian rhythm disruption. ${ }^{27}$ However, red light, lower intensity (5-10 lux) and intermittent exposure at night have 


\section{ORIGINAL ARTICLES}

Figure 1. Mean hourly light intensity data for all ICU bed areas $(A) *$ and mean \pm SEM hourly light intensity in each bed area fitted to a sin curve as per cosinor analysis (B)
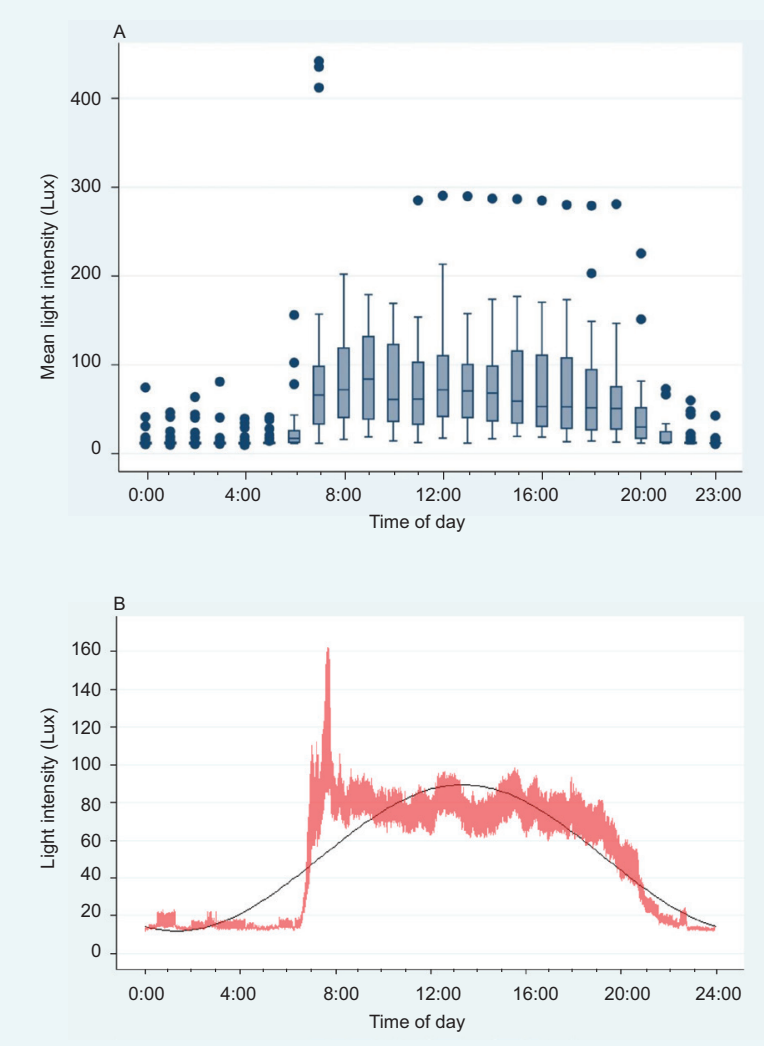

* In panel A, values shown are medians of mean values (lines within boxes), interquartile ranges (upper and lower box ends), adjacent values (whiskers) and outlying values (dots). been associated with circadian rhythm resetting. ${ }^{28}$ Artificial light exposure at night has been linked to psychological, cardiovascular and metabolic disease in addition to associations with breast cancer and prostate cancer, ${ }^{27}$ decreased muscle strength and osteoporosis. ${ }^{29}$ Extremely low frequency electric and magnetic fields have also resulted in circadian rhythm dysfunction. ${ }^{30}$ To our knowledge, only a small poorly controlled study of critically ill patients has not found bright light therapy to be effective. ${ }^{31}$

The general noise levels found in our study are similar to those found in previous studies. ${ }^{19}$ We found significant rhythm in mean sound intensity over the 24 hours of monitoring, with an amplitude of $1.5 \mathrm{~dB}$ and with sudden and unpredictable changes in sound levels relating predominantly to alarms. As humans can detect sound intensity changes by a minimum of $1 \mathrm{~dB}$, fluctuating sound intensity over a 24-hour period may function as a zeitgeber

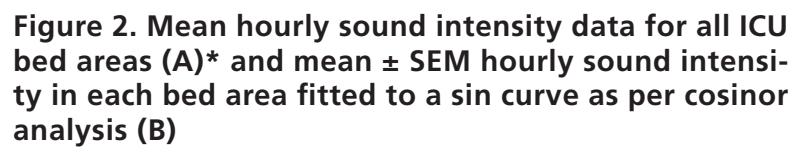

Figure 2. Mean hourly sound intensity data for all ICU bed areas $(A) *$ and mean \pm SEM hourly sound intensity in each bed area fitted to a sin curve as per cosinor analysis (B)
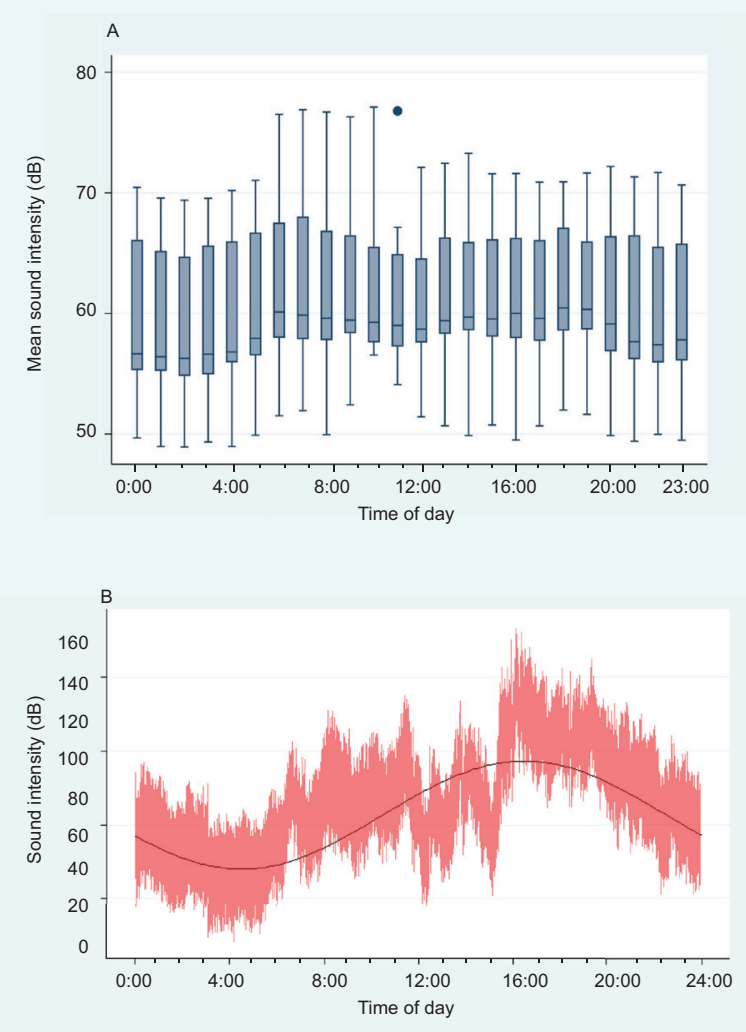

* In panel A, values shown are medians of mean values (lines within boxes), interquartile ranges (upper and lower box ends), adjacent values (whiskers) and an outlying value (dot).

in synchronising the circadian timing system. ${ }^{32}$ However, despite the observed decrease in mean sound intensity overnight, abrupt episodes of sounds greater than $100 \mathrm{~dB}$ during this time may contribute to sleep disruption and circadian dysfunction. ${ }^{33}$

In our study, diurnal variation in humidity and temperature, and significant differences between individual bed areas, were observed across the ICU despite these parameters being centrally controlled. Temperature and humidity have been documented to have detrimental effects on sleep quality, with optimal ranges of $18-22^{\circ} \mathrm{C}$ and $40-60 \%$, respectively. ${ }^{34,35}$ Although mean whole of unit values were close to ideal, individual bed areas were not assured to have optimal ambient conditions. Independent patients would fine-tune these parameters with manipulation of bed linen, but ICU patients are often unable to adjust their microenvironment. Also, while management of burns 
Figure 3. Mean hourly ambient temperature data for all ICU bed areas $(A) *$ and mean \pm SEM hourly ambient temperature in each bed area fitted to a sin curve as per cosinor analysis (B)
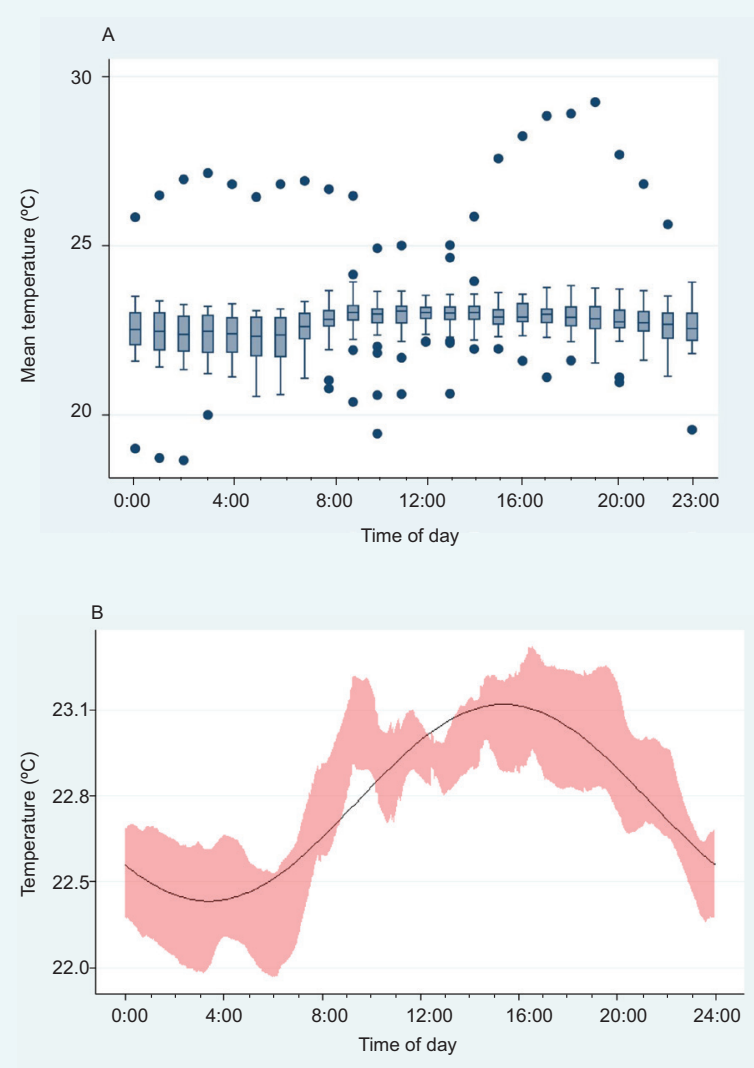

* In panel A, values shown are medians of mean values (lines within boxes), interquartile ranges (upper and lower box ends), adjacent values (whiskers) and outlying values (dots).

patients may have contributed to significant temperature differences between individual bed areas, these differences were still significant after excluding burns patients from the analysis. The rhythms that we detected in temperature and humidity suggest a capacity to function as zeitgebers.

Assessment of circadian rhythm often requires complex and expensive temporal profile measures. ${ }^{36}$ However, rhythmic CBT profile is a marker of both circadian alignment and overall homeostatic state. ${ }^{37} \mathrm{CBT}$ is generally measured routinely in the ICU, but use of temporal temperature profile as a predictor of clinical events, therapeutic effectiveness and patient outcomes has not been defined.

No significant associations between environmental conditions, illness severity, care need intensity and temperature rhythmicity were found in univariate or multivariate regression modelling. This is most likely due to the small sample size and perhaps the varied timing
Figure 4. Mean hourly relative humidity data for all ICU bed areas $(A) *$ and mean \pm SEM hourly relative humidity in each bed area fitted to a sin curve as per cosinor analysis (B)
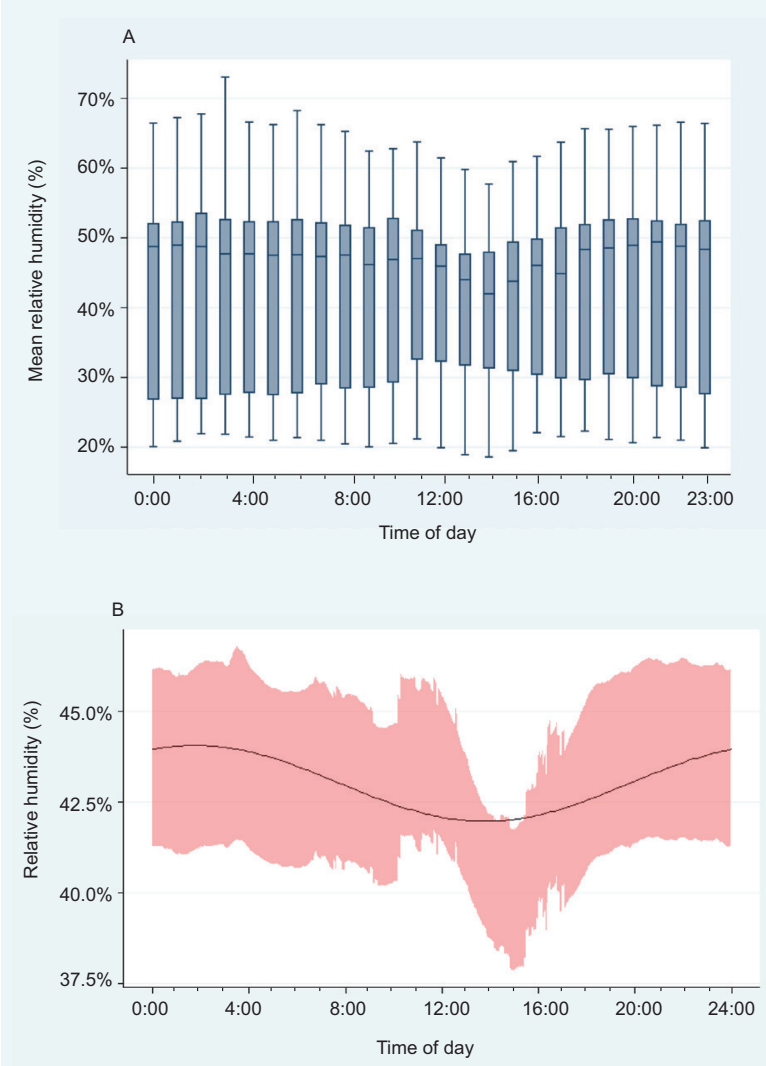

* In panel A, values shown are medians of mean values (lines within boxes), interquartile ranges (upper and lower box ends) and adjacent values (whiskers).

of surveillance during ICU stay. The ICU environment and interaction with critical illness is complex, making it difficult to assess the timing and effectiveness of interventions designed to improve patient outcomes. Such complexity includes the relationship between environment and illness severity, ${ }^{1,2}$ as well as the loss of melatonin rhythmicity in sepsis 4,38,39 and its associated anti-inflammatory and antioxidant actions. ${ }^{40} \mathrm{~A}$ high proportion of patients needed sleep-disrupting activities such as linen changes or bathing overnight in addition to frequent routine care processes of mouth care, eye care and nasogastric feeding. In addition to the effects of polypharmacy, frequent care activities contribute to disrupted rhythmicity of the sleep cycle and reduced REM sleep, resulting in a chaotic rhythm, shift in phase or insomnia.7,21,41-44 Given the crucial role of sleep as both an output and modulator of the circadian timing system, severe sleep disturbances will also have a disruptive 
Figure 5. Representative circadian comparisons of temperature data for three ICU patients (red lines and dots) versus normal temperature profiles for healthy controls (blue lines)*

A
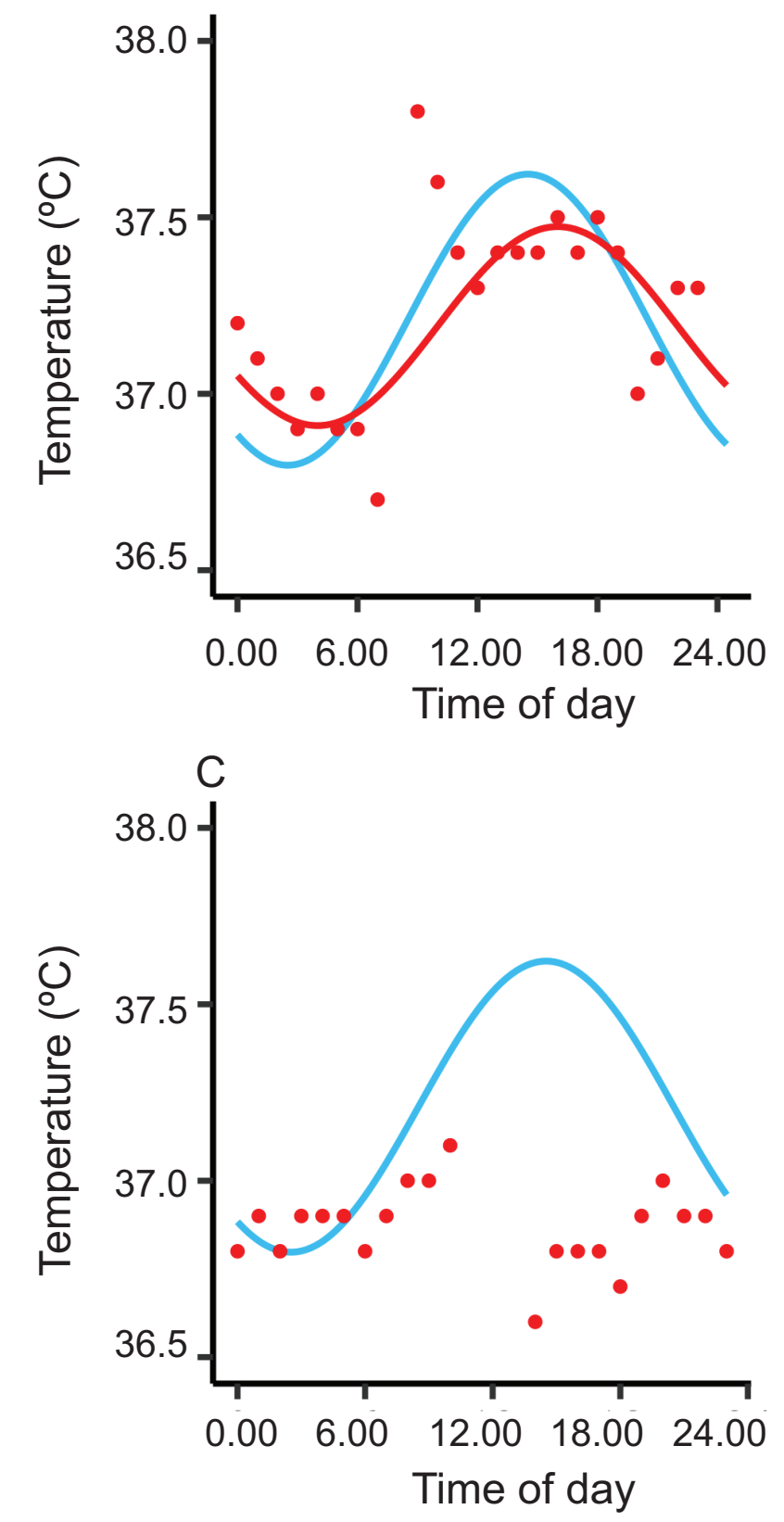

B

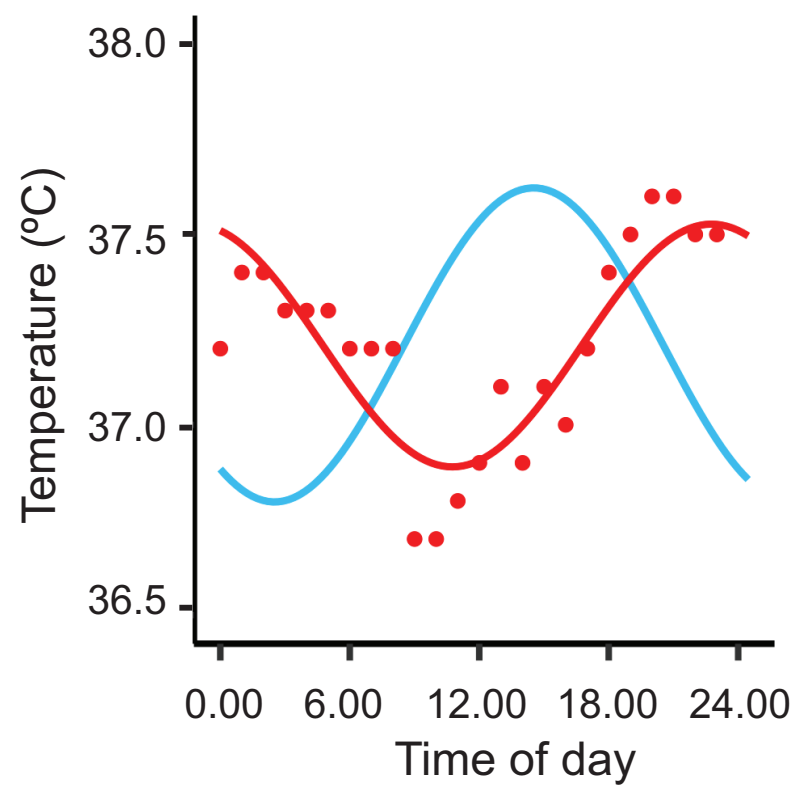

$\mathrm{ICU}=$ intensive care unit; MESOR = midline estimating statistic of rhythm. ${ }^{*}$ Panel A: Patient 5616 had no significant MESOR, amplitude or phase differences to healthy controls (rhythmicity, $P<0.001$; MESOR difference, $P=0.76$; amplitude difference, $P=0.11$; phase difference, $P=0.17$ ). Panel B: Patient 4273 had a significant phase delay and no MESOR or amplitude differences to healthy controls (rhythmicity, $P<0.001 ;$ MESOR difference, $P>0.99$; amplitude difference, $P=0.28$; phase difference, $P<0.0001$ [phase difference $=8.24$ ]). Panel $C$ : Patient 1336 had no rhythmic temperature profile (rhythmicity, $P=0.10$ ). 


\section{ORIGINAL ARTICLES}

effect on other circadian output rhythms such as those of temperature and melatonin. ${ }^{45,46}$

This report of the CHIE study provides a description of the ICU environment and its relationship with circadian health of patients as assessed by CBT profile. The study design is robust in terms of its method of environmental monitoring and linkage of ambient data to patient care activities. It uniquely uses CBT measurement as a marker of circadian health. Owing to the intensity of data collection required and the period prevalence design, the study can only provide descriptive insight into the interactions between ICU environment, patient care needs and severity of illness. The limited sample size and varied casemix restrict the power to draw inferences to inform patient care. The finding of a significant number of critically ill patients with CBT rhythmicity which may be phase advanced or delayed cautions against the uniform provision of therapies aimed at correcting circadian disturbance. However, we believe that it is an accurate representation of environmental conditions commonly experienced by ICU patients.

\section{Conclusion}

ICUs do not provide an environment in which zeitgebers can entrain a circadian rhythm. They are dark and noisy with temperature and humidity variations across bed areas. Patients receive complex pharmacotherapy. Care practices can disturb night-time sleep and do not take into account the patient's underlying chronotype or behavioural patterns such as feeding times. Despite this, a substantial number of patients have a circadian rhythm to their CBT which may be phase advanced or delayed. How these factors interact to maintain circadian health of critically ill ICU patients is not clear. Given the complexity of such interactions, the impacts of simple protocolised measures aiming to improve circadian hygiene may not be readily predictable for individual patients.

\section{Competing interests}

None declared.

\section{Author details}

Rob Boots ${ }^{1,2}$

Gabrielle Mead $^{3}$

Oliver Rawashdeh ${ }^{3}$

Judith Bellapart ${ }^{2,4}$

Shane Townsend $d^{3,4}$
Jenny Paratz $z^{2,5}$

Nicholas Garner ${ }^{3}$

Pierre Clement ${ }^{4}$

David Oddy ${ }^{4}$

On behalf of the Circadian Investigators in Critical IIIness

1 Thoracic Medicine, Royal Brisbane and Women's Hospital, Brisbane, QLD, Australia.

2 Burns, Trauma and Critical Care Research Centre, University of Queensland, Brisbane, QLD, Australia.

3 School of Biomedical Sciences, Faculty of Medicine, University of Queensland, Brisbane, QLD, Australia.

4 Department of Intensive Care Medicine, Royal Brisbane and Women's Hospital, Brisbane, QLD, Australia.

5 School of Allied Health, Griffith University, Brisbane, QLD, Australia.

Correspondence: r.boots@uq.edu.au

\section{References}

1 Fanfulla F, Ceriana P, D'Artavilla Lupo N, et al. Sleep disturbances in patients admitted to a step-down unit after ICU discharge: the role of mechanical ventilation. Sleep 2011; 34: 355-62.

2 Gabor JY, Cooper AB, Crombach SA, et al. Contribution of the intensive care unit environment to sleep disruption in mechanically ventilated patients and healthy subjects. Am J Respir Crit Care Med 2003; 167: 708-15.

3 Gehlbach BK, Chapotot F, Leproult R, et al. Temporal disorganization of circadian rhythmicity and sleep-wake regulation in mechanically ventilated patients receiving continuous intravenous sedation. Sleep 2012; 35: 1105-14.

4 Telias I, Wilcox ME. Sleep and circadian rhythm in critical illness. Critical Care 2019; 23: 82.

5 Shilo L, Dagan Y, Smorjik Y, et al. Patients in the intensive care unit suffer from severe lack of sleep associated with loss of normal melatonin secretion pattern. Am J Med Sci 1999; 317: 278-81.

6 Freedman NS, Gazendam J, Levan L, et al. Abnormal sleep/wake cycles and the effect of environmental noise on sleep disruption in the intensive care unit. Am J Respir Crit Care Med 2001; 163: 451-7.

7 Trompeo AC, Vidi Y, Locane MD, et al. Sleep disturbances in the critically ill patients: role of delirium and sedative agents. Minerva Anestesiol 2011; 77: 604-12.

8 Tamburri LM, DiBrienza R, Zozula R, Redeker NS. Nocturnal care interactions with patients in critical care units. Am J Crit Care 2004; 13: 102-12.

9 Chan M-C, Spieth PM, Quinn K, et al. Circadian rhythms: from basic mechanisms to the intensive care unit. Critical Care Med 2012; 40: 246-53.

10 im ek EM, Solak Grassie S, Emre C, Gevrek SC. Relationship between environmental conditions and nosocomial infection rates 


\section{ORIGINAL ARTICLES}

in intensive care unit. Med J Islamic World Acad Sci 2017; 25: 15-8.

11 Darbyshire JL, Young JD. An investigation of sound levels on intensive care units with reference to the WHO guidelines. Crit Care 2013; 17: R187.

12 Tainter CR, Levine AR, Quraishi SA, et al. Noise levels in surgical ICUs are consistently above recommended standards. Critical Care Med 2016; 44: 147-52.

13 Elbaz M, Leger D, Sauvet $F$, et al. Sound level intensity severely disrupts sleep in ventilated ICU patients throughout a 24 -h period: a preliminary 24-h study of sleep stages and associated sound levels. Ann Intensive Care 2017; 7: 25.

14 Fan EP, Abbott SM, Reid KJ, et al. Abnormal environmental light exposure in the intensive care environment. J Critical Care 2017; 40: 11-4.

15 Charlson ME, Pompei P, Ales KL, MacKenzie CR. A new method of classifying prognostic comorbidity in longitudinal studies: development and validation. J Chronic Dis 1987; 40: 373-83.

16 Knaus WA, Draper EA, Wagner DP, Zimmerman JE. APACHE II: a severity of disease classification system. Critical Care Med 1985; 13: 818-29.

17 Vincent JL, Moreno R, Takala J, et al. The SOFA (sepsis-related organ failure assessment) score to describe organ dysfunction/failure. On behalf of the Working Group on Sepsis-Related Problems of the European Society of Intensive Care Medicine. Intensive Care Med 1996; 22: 707-10.

18 Reis Miranda D, de Rijk A, Schaufeli W. Simplified Therapeutic Intervention Scoring System: the TISS-28 items - results from a multicenter study. Crit Care Med 1996; 24: 64-73.

19 Delaney LJ, Currie MJ, Huang H-CC, et al. The nocturnal acoustical intensity of the intensive care environment: an observational study. J Intensive Care 2017; 5: 41.

20 Moore RY. Circadian rhythms: basic neurobiology and clinical applications. Annu Rev Med 1997; 48: 253-66.

21 Kim P, Oster H, Lehnert $\mathrm{H}$, et al. Coupling the circadian clock to homeostasis: the role of period in timing physiology. Endocr Rev 2019; 40: 66-95.

22 Duffy JF, Czeisler CA. Effect of light on human circadian physiology. Sleep Med Clin 2009; 4: 165-77.

23 Olofsson K, Alling C, Lundberg D, Malmros C. Abolished circadian rhythm of melatonin secretion in sedated and artificially ventilated intensive care patients. Acta Anaesthesiol Scand 2004; 48: 679-84.

24 Reme CE, Wirz-Justice A, Terman M. The visual input stage of the mammalian circadian pacemaking system: I. Is there a clock in the mammalian eye? J Biol Rhythms 1991; 6: 5-29.

25 Spitschan M, Stefani O, Blattner $P$, et al. How to report light exposure in human chronobiology and sleep research experiments. Clocks Sleep 2019; 1: 280-9.

26 Blume C, Garbazza C, Spitschan M. Effects of light on human circadian rhythms, sleep and mood. Somnologie 2019; 23: 147-56.

27 Cho YM, S-H Ryu, Lee BR, et al. Effects of artificial light at night on human health: a literature review of observational and experimental studies applied to exposure assessment. Chronobiol Int 2015; 32: 1294-310.
28 Tahkamo L, Partonen T, Pesonen AK. Systematic review of light exposure impact on human circadian rhythm. Chronobiol Int 2019; 36: 151-70.

29 Lucassen EA, Coomans $C P$, van Putten $M$, et al. Environmental 24hr cycles are essential for health. Curr Biol 2016; 26: 1843-53.

30 Reiter RJ. Alterations of the circadian melatonin rhythm by the electromagnetic spectrum: a study in environmental toxicology. Regul Toxicol Pharmacol 1992; 15: 226-44.

31 Perras B, Meier M, Dodt C. Light and darkness fail to regulate melatonin release in critically ill humans. Intensive Care Med 2007; 33: 1954-8.

32 Belin P, McAdams S, Smith B, et al. The functional anatomy of sound intensity discrimination. J Neuroscience 1998; 18: 6388.

33 Xie H, Kang J, Mills GH. Clinical review: the impact of noise on patients' sleep and the effectiveness of noise reduction strategies in intensive care units. Critical Care 2009; 13: 208.

34 Obradovich N, Migliorini R, Mednick SC, Fowler JH. Nighttime temperature and human sleep loss in a changing climate. Science Adv 2017; 3: e1601555.

35 Okamoto-Mizuno K, Mizuno K. Effects of thermal environment on sleep and circadian rhythm. J Physiol Anthropol 2012; 31: 14.

36 Van Dycke KC, Pennings JL, van Oostrom CT, et al. Biomarkers for circadian rhythm disruption independent of time of day. PLoS One 2015; 10: e0127075.

37 McKenna HT, Reiss IK, Martin DS. The significance of circadian rhythms and dysrhythmias in critical illness. J Intensive Care 2017; 18: 121-9.

38 Mundigler G, Delle-Karth G, Koreny M, et al. Impaired circadian rhythm of melatonin secretion in sedated critically ill patients with severe sepsis. Critical Care Med 2002; 30: 536-40.

39 Sertaridou EN, Chouvarda IG, Arvanitidis KI, et al. Melatonin and cortisol exhibit different circadian rhythm profiles during septic shock depending on timing of onset: a prospective observational study. Ann Intensive Care 2018; 8: 118.

40 Tan DX, Manchester LC, Terron MP, et al. One molecule, many derivatives: a never-ending interaction of melatonin with reactive oxygen and nitrogen species? J Pineal Res 2007; 42: 28-42.

41 Telias I, Wilcox ME. Sleep and circadian rhythm in critical illness. Critical Care 2019; 23: 82.

42 Wilcox ME, Lim AS, Pinto R, et al. Sleep on the ward in intensive care unit survivors: a case series of polysomnography. Intern Med J 2018; 48: 795-802.

43 Hardin KA, Seyal M, Stewart T, Bonekat HW. Sleep in critically ill chemically paralyzed patients requiring mechanical ventilation. Chest 2006; 129: 1468-77.

44 Cooper AB, Thornley KS, Young GB, et al. Sleep in critically ill patients requiring mechanical ventilation. Chest 2000; 117: 809-18.

45 Wyatt JK, Cecco AR-D, Czeisler CA, Dijk D-J. Circadian temperature and melatonin rhythms, sleep, and neurobehavioral function in humans living on a 20-h day. Am J Physiol Regul Integr Comp Physio 1999; 277: R1152-63.

46 LeGates TA, Fernandez DC, Hattar S. Light as a central modulator of circadian rhythms, sleep and affect. Nat Rev Neurosci 2014; 15: 443. 